\title{
INTEGRAL FORMULAS FOR GLOSED SUBMANIFOLDS OF A RIEMANNIAN MANIFOLD
}

\author{
C. C. HSIUNG, JONG DIING LIU \& SITANSU S. MITTRA
}

Dedicated to Professor Buchin Su on his 57th birthday

\section{Introduction}

In 1903, H. Minkowski [11] obtained the following two integral formulas for a closed convex surface $S$ in a Euclidean 3-space $E^{3}$ :

$$
\int_{S}(1+p H) d V=0, \quad \int_{S}(H+p K) d V=0
$$

where $H$ and $K$ are respectively the mean curvature and the Gaussian curvature of $S$ at a point $P$ whose position vector with respect to the origin 0 of $E^{3}$ is $x$, $d V$ is the area element of $S$ at $P$, and $p$ is the scalar product $\langle x, e\rangle$ of $x$ and the unit normal vector $e$ of $S$ at $P$. In 1954 C. C. Hsiung [5] extended formulas (1.1) to a closed oriented hypersurface $M^{m}$ in a Euclidean $(m+1)$-space $E^{m+1}$ $(m \geq 2)$ and obtained characterizations of hyperspheres in $E^{m+1}$. In 1956 C. C. Hsiung [6] and in 1959 G. F. Feeman and C. C. Hsiung [3] extended Hsiung's integral formulas to the case in which $E^{m+1}$ is a Riemannian space $N^{m+1}$ of constant sectional curvature, and obtained characterizations of umbilical hypersurfaces in $N^{m+1}$. In 1962, Y. Katsurada [7] extended the aforesaid results to a closed oriented hypersurface in $N^{m+1}$ by introducting an infinitesimal conformal vector field $\xi$ to replace the position vector field $x$. In 1968 and 1969, Y. Katsurada, H. Kôjyô and T. Nagai [8], [9], [10] obtained integral formulas for a closed oriented submanifold $M^{m}$ of dimension $m(\geq 2)$ in a Riemannian $n$-manifold $N^{n}(n>m)$ of constant sectional curvature with respect to an infinitesimal conformal vector field $\xi$ and a special unit normal vector field $e$ of $M^{m}$, and conditions for $M^{m}$ to be umbilical with respect to $e$. In 1971 B. Y. Chen and K. Yano [1] studied the case in which the field $e$ is more general but $N^{n}$ is Euclidean and $\xi$ is the position vector field $x$. The purpose of the present paper is to extend the results of Chen and Yano to the general case in which $N^{n}$ is Riemannian and $\xi$ is an infinitesimal conformal vect or field so that all known results are special cases of ours.

Communicated April 19, 1972, and, in revised form, May 28, 1976. The work of the second author was done during his visit to Lehigh University and partially supported by the National Science Council of the Republic of China. 
In $\S 2$ we first define the vector product of two tangent vectors of a Riemannian $n$-manifold $N^{n}$ at a point $P$, and then discuss orthonormal frames $P e_{i_{1}} e_{i_{2}} \cdots e_{i_{n}}$ on $N^{n}$ at $P$.

$\S 3$ contains the fundamental definitions and formulas for a submanifold $M^{m}$ of dimension $m(\geq 2)$ immersed in $N^{n}(n>m)$. In particular, some formulas are reduced to simpler forms when $N^{n}$ is of constant sectional curvature.

Suppose that $N^{n}$ admits a continuous infinitesimal conformal vector field $\xi$, and let $e$ be a unit normal vector field over $M^{m}$ parallel in the normal bundle of $M^{m}$. In $\S 4$ we derive integral formulas for a closed oriented $M^{m}$ in $N^{n}$ with respect to $\xi$ and $e$, and in $\S 5$ we obtain various conditions for $M^{m}$ to be umbilical with respect to $e$.

We wish to thank Y. Katsurada for her discussion with one of us about some computation involving the infinitesimal conformal vector field $\xi$.

\section{Vector product and orthonormal frames}

Throughout this paper unless stated otherwise the ranges of indices are given as follows :

$$
\begin{aligned}
1 & \leq i, j, k, \cdots \leq m, \\
1 & \leq \alpha, \beta, \gamma, \cdots \leq n, \\
m+1 & \leq A, B, C, \cdots \leq n, \quad(m<n) .
\end{aligned}
$$

We shall also follow the usual tensor convention that when a letter appears in any term as a subscript and a superscript, it is understood that this letter is summed over its range.

Let $N^{n}$ be a Riemannian manifold of dimension $n(\geq 3)$ and class $C^{3}$, $\left(x^{1}, \cdots, x^{n}\right)$ local coordinates of a point $P$ in $N^{n}$, and $a_{\alpha \beta} d x^{\alpha} d x^{\beta}$ a Riemannian metric of $N^{n}$, where $a_{\alpha \beta}=a_{\beta \alpha}$ and the matrix $\left(a_{\alpha \beta}\right)$ is positive definite so that the determinant $\left|a_{\alpha \beta}\right|=a$ is positive.

Let $A_{1}, \cdots, A_{n-1}$ be $n-1$ tangent vectors of the manifold $N^{n}$ at the point $P$, and $A_{i}^{\alpha}$ the contravariant components of $A_{i}$ in the local coordinate system $x^{1}, \cdots, x^{n}$. Let $A_{1} \times \cdots \times A_{n-1}$ denote the vector product of the $n-1$ vectors $A_{1}, \cdots, A_{n-1}$, which is defined to be the tangent vector of the manifold $M^{n}$ at $P$ whose $\beta$-th contravariant component is (see, for instance, Feeman and Hsiung [3])

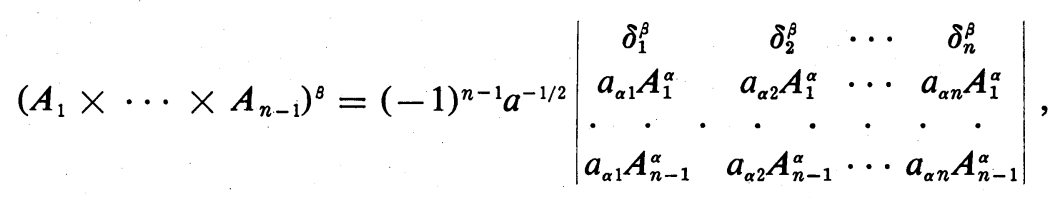

where $\delta_{\alpha}^{\beta}$ are the Kronecker deltas. Let $T$ be a tangent vector of the manifold $N^{n}$ at the point $P$ with contravariant components $T^{\alpha}$ in $x^{1}, \cdots, x^{n}$. From the 
definition of the scalar product of any two vectors $A_{i}$ and $A_{j}$, namely,

$$
\left\langle A_{i}, A_{j}\right\rangle=a_{\alpha \beta} A_{i}^{\alpha} A_{j}^{\beta},
$$

it follows that the scalar product of the two vectors $T$ and $A_{1} \times \cdots \times A_{n-1}$ is given by

$$
\left\langle T, A_{1} \times \cdots \times A_{n-1}\right\rangle=(-1)^{n-1} a^{1 / 2}\left|T, A_{1}, \cdots, A_{n-1}\right|,
$$

where $\left|T, A_{1}, \cdots, A_{n-1}\right|$ is a determinant, the elements of each of whose columns are the contravariant components of the vector indicated. Thus by (2.4) it is readily seen that the vector $A_{1} \times \cdots \times A_{n-1}$ is orthogonal to each of the $n-1$ vectors $A_{1}, \cdots, A_{n-1}$.

Now consider an orthonormal frame $P e_{1} \cdots e_{n}$ on $N^{n}$ at $P$, where $e_{1}, \cdots, e_{n}$ form an ordered set of $n$ mutually orthogonal unit tangent vectors of the manifold $N^{n}$ at $P$ so that

$$
\left\langle e_{\alpha}, e_{\beta}\right\rangle=a_{\gamma \dot{\delta}} e_{\alpha}^{\gamma} e_{\beta}^{\delta}=\delta_{\alpha \beta},
$$

where $\delta_{\alpha \beta}$ are the Kronecker deltas. The position vector $x$ of the point $P$ is defined to be the tangent vector of the manifold $N^{n}$ at the point $P$ whose contravariant components are the local coordinates $x^{1}, \cdots, x^{n}$ of the point $P$.

Let $\alpha_{1}, \cdots, \alpha_{n}$ be distinct and suppose that $1 \leq \alpha_{1}, \cdots, \alpha_{n} \leq n$. Then we can write

$$
e_{\alpha_{1}} \times \cdots \times e_{\alpha_{n-1}}=c e_{\alpha_{n}},
$$

where $c$ is a function of the $x$ 's. In order to find an expression for $c$, we consider the two matrices

$$
\phi=\left(\phi_{\beta}^{i}\right), \quad \psi=\left(\psi_{i}^{\beta}\right), \quad(i=1, \cdots, n-1),
$$

where

$$
\phi_{\beta}^{i}=a_{\alpha \beta} e_{i}^{\alpha}, \quad \psi_{i}^{\beta}=e_{i}^{\beta},
$$

the superscript of the element $\phi_{\beta}^{i}$ or $\psi_{i}^{\beta}$ indicating the row to which the element belongs, and the subscript indicating the column. From (2.2) and (2.6) it is easily seen that

$$
c e_{\alpha_{n}}^{r}=(-1)^{n+r} B^{r} a^{-1 / 2}, \quad(\gamma=1, \cdots, n),
$$

where $B^{r}$ is the determinant of the matrix of $(n-1)$ th order obtained by deleting the $\gamma$-th column from the matrix $\phi$. Substitution of (2.9) in (2.5) for $\alpha=$ $\beta=\alpha_{n}$ gives 


$$
c^{2}=B
$$

where

$$
B=\left|\begin{array}{cccc}
B^{1} & -B^{2} & \cdots & (-1)^{n-1} B^{n} \\
e_{\alpha_{1}}^{1} & e_{\alpha_{1}}^{2} & \cdots & e_{\alpha_{1}}^{n} \\
\cdot & \cdot & \cdot & \cdot \\
e_{\alpha_{n-1}}^{1} & e_{\alpha_{n-1}}^{2} & \cdots & e_{\alpha_{n-1}}^{n}
\end{array}\right|
$$

which is equal to the sum of the products of the corresponding determinants of the $(n-1)$ th order of the two matrices (2.7). By an elementary theorem on determinants (see, for instance, [2, p. 102]), from (2.5) it follows immediately that

$$
B=\left|\phi_{\beta}^{i} \psi_{i}^{\beta}\right|=1,
$$

which, together with (2.10), implies that

$$
c= \pm 1 \text {. }
$$

If the orientations of $e_{1}, \cdots, e_{n}$ are so chosen that

$$
\left|e_{1}, \cdots, e_{n}\right|>0
$$

then by taking the scalar product of the vector $e_{\alpha_{n}}$ with each side of (2.6) and using (2.4), (2.13), we can easily obtain

$$
\left|e_{1}, \cdots, e_{n}\right|=a^{-1 / 2},
$$

and therefore

$$
e_{\alpha_{1}} \times \cdots \times e_{\alpha_{n-1}}=\delta_{\alpha_{1} \cdots \alpha_{n}} e_{\alpha_{n}},
$$

where $\delta_{\alpha_{1} \cdots \alpha_{n}}=+1$ or -1 according as the permutation of $\alpha_{1}, \cdots, \alpha_{n}$ into $1, \cdots, n$ is even or odd.

\section{Immersed submanifolds}

Let $x: M^{m} \rightarrow N^{n}$ be an $m$-dimensional $(2 \leq m<n)$ submanifold of class $C^{3}$ immersed in a Riemannian $n$-manifold $N^{n}$ defined in $\S 2$. For simplicity we shall write $x\left(M^{m}\right)$ as $M^{m}$. Let $\left(u^{1}, \cdots, u^{m}\right)$ be local coordinates of a point $P$ on $M^{m}$. Then

$$
x^{\alpha}=x^{\alpha}\left(u^{1}, \cdots, u^{m}\right), \quad(\alpha=1, \cdots, n),
$$

are of class $C^{3}$, and the first fundamental form of $M^{m}$ at $P$ is defined to be 


$$
I=\langle d x, d x\rangle=g_{i j} d u^{i} d u^{j},
$$

where $d$ denotes the exterior differentiation, and the matrix $\left(g_{i j}\right)$ is positive definite so that the determinant $\left|g_{i j}\right|=g>0$. Let $x_{, i}^{\alpha}$ denotes the covariant derivative of $x^{\alpha}$ with respect to $g_{i j}$. Then it is known that

$$
\begin{gathered}
x_{, i}^{\alpha}=\partial x^{\alpha} / \partial u^{i}, \\
g_{i j}=a_{\alpha \beta} x_{i,}^{\alpha} x_{, j}^{\beta} .
\end{gathered}
$$

The element of volume of $M^{m}$ at $P$ is given by

$$
d V=\sqrt{g} d u^{1} \wedge \cdots \wedge d u^{m},
$$

where $\wedge$ denotes the exterior multiplication.

Now we are in a position to introduce the generalized covariant differentiation, which is useful for studying submanifolds of Riemannian manifolds. Let $A_{\beta i}^{\alpha}$ be a mixed tensor of the second order in the $x$ 's and a covariant vector in the $u$ 's, as indicated by the Greek and Latin indices. Then following A. W. Tucker [13], the generalized covariant derivative of $A_{\beta i}^{\alpha}$ with respect to the $u$ 's is defined by

$$
\nabla_{j} A_{\beta i}^{\alpha}=\partial A_{\beta i}^{\alpha} / \partial u^{j}+\Gamma_{\gamma \delta}^{\alpha} A_{\beta i}^{\gamma} x_{, j}^{\delta}-\Gamma_{\beta \delta}^{\gamma} A_{\gamma i}^{\alpha} x_{, j}^{\delta}-\Gamma_{i j}^{k} A_{\beta k}^{\alpha},
$$

where the Christoffel symbols $\Gamma_{\beta r}^{\alpha}$ with Greek indices are formed with respect to the $a_{\alpha \beta}$ and the $x$ 's as follows:

$$
\Gamma_{\beta \gamma}^{\alpha}=\frac{1}{2} a^{\alpha \delta}\left(\frac{\partial a_{\beta \delta}}{\partial x^{\tau}}+\frac{\partial a_{\gamma \delta}}{\partial x^{\beta}}-\frac{\partial a_{\beta \gamma}}{\partial x^{\delta}}\right),
$$

$\left(a^{\alpha \beta}\right)$ being the inverse matrix of $\left(a_{\alpha \beta}\right)$, and those $\Gamma_{j k}^{i}$ with Latin indices are formed with respect to the $g_{i j}$ and the $u$ 's in a similar way. It should be noted that this definition of generalized covariant differentiation can be applied to any tensor in the $u$ 's and the $x$ 's, and that the generalized covariant differentiation of sums and products of tensors obeys the ordinary rules. If a tensor is one with respect to the $u$ 's only, so that only Latin indices appear, its generalized covariant derivative is the same as its covariant derivative with respect to the $u$ 's. Furthermore, in generalized covariant differentiation, the fundamental tensors $a_{\alpha \beta}$ and $g_{i j}$ can be treated as constants. Since $x^{\alpha}$ is an invariant for the transformation of $u$ 's, its generalized covariant derivative is the same as its covariant derivative with respect to the $u$ 's, so that

$$
\nabla_{i} x^{\alpha}=x_{, i}^{\alpha}=\partial x^{\alpha} / \partial u^{i} .
$$

At a point $P$ on $M^{m}$ we can choose $e_{m+1}, \cdots, e_{n}$ of the orthonormal frame $P e_{1} \cdots e_{n}$ on $N^{n}$ defined in $\S 2$ to be unit normal vectors of $M^{m}$. Then we can have (see, for instance, [16, Chapter X]) 


$$
\begin{gathered}
\nabla_{i} x_{, j}=\sum_{A} \Omega_{A \mid i j} e_{A}, \\
\Omega_{A \mid i j}=\left\langle\nabla_{j} x_{, i}, e_{, A}\right\rangle, \\
\nabla_{i} e_{A}=-\Omega_{A \mid i k} g^{k j} x_{, j}+\sum_{B} \vartheta_{B A \mid i} e_{B},
\end{gathered}
$$

where $\left(g^{i j}\right)$ is the inverse matrix of $\left(g_{i j}\right)$, and

$$
\begin{gathered}
\Omega_{A \mid i j}=\Omega_{A \mid j i}, \\
\vartheta_{A B \mid i}+\vartheta_{B A \mid i}=0,
\end{gathered}
$$

so that $\vartheta_{A A \mid i}=0$. Thus being defined to be $-\left\langle d x, d e_{A}\right\rangle$ the second fundamental form $I I_{A}$ of $M^{m}$ with respect to $e_{A}$ is given by

$$
I I_{A}=\Omega_{A \mid i j} d u^{i} d u^{j} .
$$

The equations of Gauss and Mainardi-Codazzi of $M^{m}$ in $N^{n}$ are (see, for instance, [2, p. 162])

$$
\begin{gathered}
R_{h i j k}=\sum_{A}\left(\Omega_{A \mid h k} \Omega_{A \mid i j}-\Omega_{A \mid h j} \Omega_{A \mid i k}\right)+\bar{R}_{\alpha \beta \gamma \delta} x_{, h}^{\alpha} x_{, i}^{\beta} x_{, j}^{\gamma} x_{, k}^{x^{\gamma}}, \\
\Omega_{C \mid i j, k}-\Omega_{C \mid i k, j}=\sum_{B}\left(\vartheta_{B C \mid k} \Omega_{B \mid i j}-\vartheta_{B C \mid j} \Omega_{B \mid i k}\right) \\
+\bar{R}_{\alpha \beta \gamma \delta} d_{C}^{\alpha} x_{, i}^{\beta} x_{, j}^{\gamma} x_{, k}^{\delta},
\end{gathered}
$$

where the Riemann symbols $R_{h i j k}=g_{h l} R^{l}{ }_{i j k}$ for $M^{m}$ formed with respect to the $g_{i j}$ and the $u$ 's are defined by

$$
R_{i j k}^{h}=\frac{\partial \Gamma_{i j}^{h}}{\partial u^{k}}-\frac{\partial \Gamma_{i k}^{h}}{\partial u^{j}}+\Gamma_{i j}^{l} \Gamma_{l k}^{h}-\Gamma_{i k}^{l} \Gamma_{l j}^{h},
$$

and the Riemann symbols $\bar{R}_{\alpha \beta \gamma \delta}$ for $N^{n}$ formed with respect to the $a_{\alpha \hat{\beta}}$ and the $x$ 's can be similarly defined.

In particular, if the manifold $N^{n}$ is of constant sectional curvature $C$, from the definition it follows that

$$
\bar{R}_{\alpha \beta \gamma \delta}=C\left(a_{\alpha \delta} a_{\beta \gamma}-a_{\alpha \gamma} a_{\beta \delta}\right),
$$

and therefore $(3.15),(3.16)$ are reduced, in consequence of (3.4), to

$$
\begin{gathered}
R_{h i j k}=\sum_{A}\left(\Omega_{A \mid h k} \Omega_{A \mid i j}-\Omega_{A \mid h j} \Omega_{A \mid i k}\right)+C\left(g_{h k} g_{i j}-g_{h j} g_{i k}\right) \\
\Omega_{C \mid i j, k}-\Omega_{C \mid i k, j}=\sum_{B}\left(\vartheta_{B C \mid k} \Omega_{B \mid i j}-\vartheta_{B C \mid j} \Omega_{B \mid i k}\right)
\end{gathered}
$$

Moreover, by using (3.11), (3.9), (3.20) we can easily obtain 


$$
\begin{aligned}
d^{2} e_{A} & =d\left(\nabla_{i} e_{A} d u^{i}\right) \\
& =\sum_{B}\left(\nabla_{l} \vartheta_{B A \mid i}-\Omega_{A \mid i k} \Omega_{B \mid l j} g^{k j}+\sum_{C} \vartheta_{C A \mid i} \vartheta_{B C \mid l}\right) e_{B} d u^{l} \wedge d u^{i}
\end{aligned}
$$

The principal curvature of $M^{m}$ at $P$ with respect to a normal vector $e_{A}$ $(m+1 \leq A \leq n)$ are the eigenvalues $k_{1}\left(e_{A}\right), \cdots, k_{m}\left(e_{A}\right)$ of the matrix $\left(\Omega_{A \mid i j}\right)$ relative to the matrix $\left(g_{i j}\right)$, i.e., the roots of the determinant equation

$$
\operatorname{det}\left(\Omega_{A \mid i j}-\lambda g_{i j}\right)=0
$$

in $\lambda$, and the $r$ th mean curvature of $M^{m}$ at $P$ with respect to $e_{A}$ is defined to be the $r$ th elementary symmetric function of $k_{1}\left(e_{A}\right), \cdots, k_{m}\left(e_{A}\right)$ divided by the number of terms, i.e.,

$$
\begin{aligned}
\left(\begin{array}{c}
m \\
r
\end{array}\right) K_{r}\left(e_{A}\right)=\sum_{i_{1}<\cdots<i_{r}} k_{i_{1}}\left(e_{A}\right) \cdots k_{i_{r}}\left(e_{A}\right), \\
\\
\quad(1 \leq r \leq m, m+1 \leq A \leq n),
\end{aligned}
$$

where $\left(\begin{array}{c}m \\ r\end{array}\right)=m ! /(r !(m-r) !)$. For convenience, we assume that $K_{0}\left(e_{A}\right)=$ $=1$.

$P \in M^{m}$ is called an umbilical point of $M^{m}$ with respect to $e_{A}$ if $k_{1}\left(e_{A}\right)=$ $\cdots=k_{m}\left(e_{A}\right)$ at $P$, and $M^{m}$ is called an umbilical submanifold of $N^{n}$ with respect to a vector field $e_{A}$ if every point of $M^{m}$ is an umbilical point with respect to $e_{A}$ at that point. It is well known that a closed oriented hypersurface in a Euclidean space $E^{m+1}$ consisting entirely of umbilical points with respect to the unique normal vector field is a hypersphere.

If $k_{a}$ is a real simple root of (3.22), then

$$
\left.\left(\Omega_{A i i j}-k_{a} g_{i j}\right) p_{a}\right|^{i}=0, \quad(j=1, \cdots, m),
$$

define, to within a factor, $m$ quantities $\left.p_{a}\right|^{i}, i=1, \cdots, m$, which are the contravariant components of a real vector in the tangent space of $M^{m}$ at $P$, called a principal vector of $M^{m}$ at $P$ corresponding to the principal curvature $k_{a}$, as is seen by changing the coordinates and making use of the tensor properties of $\Omega_{A \mid i j}$ and $g_{i j}$. If $k_{b}$ is another real simple root of (3.22), we have a second vector $\left.p_{b}\right|^{i}$ defined by

$$
\left.\left(\Omega_{A i i j}-k_{b} g_{i j}\right) p_{b}\right|^{i}=0, \quad(j=1, \cdots, m) .
$$

Multiplying (3.24) by $\left.p_{b}\right|^{j}$ and (3.25) by $\left.p_{\alpha}\right|^{j}$, summing for $j$ in each case and subtracting, we have, since $k_{a} \neq k_{b}$ by hypothesis,

$$
\left.\left.g_{i j} p_{a}\right|^{i} p_{b}\right|^{j}=0
$$


that is, the two vectors $\left.p_{a}\right|^{i}$ and $\left.p_{b}\right|^{j}$ are orthogonal. Hence, as is well known, the $m$ principal vectors $\left.p_{1}\right|^{i}, \cdots,\left.p_{m}\right|^{i}$ corresponding to the $m$ principal curvatures $k_{1}, \cdots, k_{m}$ with respect to the unit normal vector $e_{A}$ of $M^{m}$ at $P$ are mutually orthogonal.

Lemma 3.1. By a suitable choice of the local coordinates $u^{1}, \cdots, u^{m}$ of $M^{m}$ at a point $P$ we have

$$
\nabla_{i} e_{A}=-k_{i} x_{, i}+\sum_{B} \vartheta_{B A \mid i} e_{B}, \quad(i=1, \cdots, m, \text { not summed }),
$$

where $k_{1}, \cdots, k_{m}$ are the principal curvatures of $M^{m}$ at $P$ with respect to $e_{A}$.

Proof. Choose the local coordinates $u^{1}, \cdots, u^{m}$ of $M^{m}$ at $P$ such that $x_{, 1}$, $\cdots, x_{, m}$ to be the $m$ principal vectors $\left.p_{1}\right|^{i}, \cdots,\left.p_{m}\right|^{i}$ of $M^{m}$ at $P$ corresponding to $k_{1}, \cdots, k_{m}$, so that $g_{i j}=0$ for $i \neq j$ at $P$. The contravariant components $x_{, a}^{\alpha}$ and $\left.p_{a}\right|^{i}$ of the principal vector $p_{a} \mid$ in the $x$ 's and the $u$ 's respectively are connected by the relation

$$
x_{, a}^{\alpha}=\left.x_{, i}^{\alpha} p_{a}\right|^{i}
$$

Multiplying (3.28) by $a_{\alpha \beta} x_{, b}^{\beta}$ and summing for $\alpha$ we obtain $g_{a b}=\left.g_{b i} p_{a}\right|^{i}$ from which it follows that

$$
\left.p_{a}\right|^{i}=\delta_{a}^{i}
$$

Substituting (3.29) in (3.24) gives

$$
\Omega_{A \mid i j}=k_{i} g_{i j}, \quad(i=1, \cdots, m, \text { not summed }) .
$$

From (3.30) and (3.11) follows immediately (3.27). q.e.d.

Let $\xi$ be an infinitesimal conformal vector field on the manifold $N^{n}$, and $L_{\xi}$ the Lie derivative with respect to $\xi$. Then on $N^{n}$ we have

$$
L_{\xi} a_{\alpha \beta}=\xi_{\alpha, \beta}+\xi_{\beta, \alpha}=2 \rho a_{\alpha \beta},
$$

where $\rho$ is a function of $x^{1}, \cdots, x^{n}$. The field $\xi$ is said to be homethetic or isometric according as $\rho$ is constant or zero.

Lemma 3.2. If the local coordinates $x^{1}, \cdots, x^{n}$ on $N^{n}$ are so chosen that the Kronecker vector $\delta_{1}$, whose contravariant components are the Kronecker deltas $\delta_{1}^{1}, \cdots, \delta_{1}^{n}$, generate an infinitesimal conformal vector field on $N^{n}$, then on $N^{n}$

$$
L_{\delta_{1}} a_{\alpha \beta}=2 \rho a_{\alpha \beta}=\partial a_{\alpha \beta} / \partial x^{1} .
$$

Proof. From the definition of cavariant differentiation with respect to the $x$ 's it follows that

$$
\delta_{1 \alpha, \beta}=a_{\alpha \gamma} \delta_{1, \beta}^{r}=a_{\alpha \gamma} \Gamma_{1 \beta}^{r}
$$


and similarly $\delta_{1 \beta, \alpha}=a_{\beta \gamma} \Gamma_{1 \alpha}^{r}$. By means of (3.7) we readily have $a_{\alpha \gamma} \Gamma_{1 \beta}^{r}+a_{\beta \gamma} \Gamma_{1 \alpha}^{\gamma}$ $=\partial a_{\alpha \beta} / \partial x^{1}$, which together with (3.31) gives (3.32). q.e.d.

If the vector $\delta_{1}$ generates an infinitesimal conformal vector field on $N^{n}$, then using (3.33) we immediately obtain that on $N^{n}$

$$
d\left(\delta_{1}^{\alpha}\right)=\delta_{1, \gamma}^{\alpha} d x^{\tau}=\delta_{1, \gamma}^{\alpha} x_{, i}^{\tau} d u^{i}=\Gamma_{{ }_{\gamma}}^{\alpha} x_{, i}^{\tau} d u^{i}
$$

which together with (3.7) implies

$$
a_{\alpha \beta} d \delta_{1}^{\alpha}=\frac{1}{2}\left(\frac{\partial a_{1 \beta}}{\partial x^{\gamma}}+\frac{\partial a_{r \beta}}{\partial x^{1}}-\frac{\partial a_{1 \gamma}}{\partial x^{\beta}}\right) x_{, i}^{\gamma} d u^{i}
$$

\section{Integral formulas}

Let $x: M^{m} \rightarrow N^{n}$ be an $m$-dimensional $(2 \leq m<n)$ submanifold of class $C^{3}$ immersed in a Riemannian $n$-manifold $N^{n}$, which is of constant sectional curvature and admits a continuous infinitesimal conformal vector field $\xi$, so that $\S \S 2$ and 3 can be applied. In this section we shall derive some integral formulas for closed oriented $\boldsymbol{M}^{m}$ with respect to a fixed unit normal vector field, $e_{m+1}$ say, on $M^{m}$. For this purpose we choose the orientation of the orthonormal frame $P e_{1} \cdots e_{n}$ of $N^{n}$ at a point $P$ defined in $\S 3$ such that (2.14) and therefore (2.15) hold, and we also choose the local coordinates $x^{1}, \cdots, x^{n}$ and $u^{1}, \cdots, u^{m}$ of $N^{n}$ and $M^{m}$ at $P$ respectively such that the Kronecker vector $\delta_{1}$ be the infinitesimal conformal vector $\xi$, and that $x_{, 1}, \cdots, x_{, m}$ be the $m$ principal vectors $\left.p_{1}\right|^{i}, \cdots,\left.p_{m}\right|^{i}$ of $M^{m}$ at $P$ with respect to $e_{m+1}$, so that at $P$

$$
\begin{gathered}
g_{i j}=0, \quad(i \neq j), \\
e_{i}=x_{, i} /[i], \quad(i=1, \cdots, m),
\end{gathered}
$$

where

$$
[i]=\sqrt{g_{i i}} \text {. }
$$

Now we are in a position to evaluate the following exact differential $m$-form for $1 \leq i \leq m$ :

$$
\begin{aligned}
d\left(\sqrt{a} \mid \delta_{1}\right. & \underbrace{d x, \cdots, d x}_{m-i}, \underbrace{d e_{m+1}, \cdots, d e_{m+1}}_{i-1}, e_{m+1}, \cdots, e_{n} \mid) \\
= & (\mathrm{I})+(-1)^{m-i}(i-1)(\mathrm{II})+(-1)^{m-1}(\mathrm{III}) \\
& +(-1)^{m-1}(\mathrm{IV})+(-1)^{m-1} \sum_{\alpha=3}^{n-m}(\mathrm{~V})_{\alpha}
\end{aligned}
$$

where we have used $d^{2} x=0$ and put 


$$
\text { (I) }=\sqrt{a}|d \delta_{1}, \underbrace{d x, \cdots, d x}_{m-i}, \underbrace{d e_{m+1}, \cdots, d e_{m+1}}_{i-1}, e_{m+1} \cdots, e_{n}| \text {, }
$$

$$
\begin{aligned}
& \text { (II) }=\sqrt{a}|\delta_{1}, \underbrace{d x, \cdots, d x}_{m-i}, d^{2} e_{m+1}, \underbrace{d e_{m+1}, \cdots, d e_{m+1}}_{i-2}, e_{m+1}, \cdots, e_{n}| \text {, } \\
& (\mathrm{III})=\sqrt{a}|\delta_{1}, \underbrace{d x, \cdots, d x}_{m-i}, \underbrace{d e_{m+1}, \cdots, d e_{m+1}}_{i}, e_{m+2}, \cdots, e_{n}| \text {, } \\
& (\mathrm{IV})=\sqrt{a} \mid \delta_{1}, \underbrace{d x, \cdots, d x}_{m-i}, d \underbrace{d e_{m+1}, \cdots, d e_{m+1}}_{i-1}, e_{m+1}, d e_{m+2}, \\
& e_{m+3}, \cdots, e_{n} \mid, \\
& (\mathrm{V})_{\alpha}=\sqrt{a} \mid \delta_{1}, \underbrace{d x, \cdots, d x}_{m-i}, d \underbrace{d e_{m+1}, \cdots, d e_{m+1}}_{i-1} \text {, } \\
& e_{m+1}, e_{m+2}, \cdots, d e_{m+\alpha}, \cdots, e_{n} \mid, \quad(3 \leq \alpha \leq n-m) .
\end{aligned}
$$

By means of $(3,27)$ for $A=m+1,(4.2),(4.3),(2.4),(2.3),(2.16)$, (3.35), (3.32), (3.4), (3.5), (4.1) we obtain

$$
\begin{aligned}
& \text { (I) }=(-1)^{i-1} \sqrt{a} \mid d \delta_{1},\left[j_{1}\right] e_{j_{1}} d u^{j_{1}}, \cdots,\left[j_{m-i}\right] e_{j_{m-i}} d u^{j_{m-i}} \text {, } \\
& k_{j_{m-i+1}}\left[j_{m-i+1}\right] e_{j_{m-i+1}} d u^{j_{m-i+1}}, \\
& \cdots, k_{j_{m-1}}\left[j_{m-i}\right] e_{j_{m-1}} d u^{j_{m-1}}, e_{m+1}, \cdots, e_{n} \mid \\
& =(-1)^{m+i}(m-i) !(i-1) ! a_{\alpha \beta} d \delta_{1}^{\alpha} \wedge \frac{x_{, j_{m}}^{\beta}}{\left[j_{m}\right]}\left[j_{1}\right] \cdots\left[j_{m-1}\right] \\
& \cdot \delta_{j_{1} \cdots j_{m}} k_{j_{m-i+1}} \cdots k_{j_{m-1}} d u^{j_{1}} \wedge \cdots \wedge d u^{j}{ }_{m-1} \\
& =(-1)^{n+i}(m-i) !(i-1) !\left\langle d \delta_{1},\left(\left[j_{1}\right] \cdots\left[j_{m-1}\right] e_{j_{1}}\right.\right. \\
& \text { (4.10) } \left.\quad \times \cdots \times e_{j_{m-1}} \times e_{j_{m+1}} \times \cdots \times e_{n}\right) \\
& \left.\cdot k_{j_{m-i+1}} \cdots k_{j_{m-1}} d u^{j_{1}} \wedge \cdots \wedge d u^{j_{m-1}}\right\rangle \\
& =(-1)^{m+i} \frac{(m-i) !(i-1) !}{2\left[j_{m}\right]}\left[j_{1}\right] \cdots\left[j_{m-1}\right] \frac{\partial a_{\alpha \beta}}{\partial x^{1}} x_{, j_{m}}{ } x_{, j_{m}} \\
& \cdot \delta_{j_{1} \cdots j_{m}} k_{j_{m-i+1}} \cdots k_{j_{m-1}} d u^{j_{m}} \wedge d u^{j_{1}} \wedge \cdots \wedge d u^{j_{m-1}} \\
& =(-1)^{i-1} \rho(m-i) !(i-1) !(m-i+1) \\
& \sum_{j_{m-i+1}, \cdots, j_{m-1}} k_{j_{m-i+1}} \cdots k_{j_{m-1}} d V \text {. }
\end{aligned}
$$

It should be remarked that in the summation on $j_{1}, \cdots, j_{m}$ in (4.10) for fixed $j_{m-i+1}, \cdots, j_{m-1}, m-i$ other $j$ 's are together and their order is immaterial, and the remaining $j$ can take any one of the other $(m-i+1) j$ 's, namely, $j_{1}, \cdots, j_{m-i}, j_{m}$, so that we get the factor $m-i+1$. From (4.10) and (3.23) follows immediately 


$$
\text { (I) }=(-1)^{i-1} m ! \rho K_{i-1}\left(e_{m+1}\right) d V .
$$

Substituting (3.21) for $A=m+1$ in (4.6) gives readily

$$
\text { (II) }=0 \text {. }
$$

Using the same method as above we can easily obtain

$$
\begin{aligned}
(\mathrm{III})= & (-1)^{m+i} m !\left\langle\delta_{1}, e_{m+1}\right\rangle K_{i}\left(e_{m+1}\right) d V, \\
(\mathrm{IV})= & (-1)^{m+i}(m-i) !(i-1) !\left\langle\delta_{1}, e_{m+2}\right\rangle \\
& \cdot \sum_{j_{m-i+1}, \cdots, j_{m}} k_{j_{m-i+1}} \cdots k_{j_{m-1}} \Omega_{m+2 \mid j k} g^{k_{m}} d V .
\end{aligned}
$$

The vector field $\delta_{1}$ can be decomposed into two parts :

$$
\delta_{1}=\delta_{1 \mid t}+\delta_{1 \mid n}
$$

where $\delta_{1 \mid t}$ is tangent to $M^{m}$, and $\delta_{1 \mid n}$ normal to $M^{m}$. Let $e$ and $\bar{e}$ be two unit normal vector fields over $M^{m}$ coplanar with $\delta_{1 \mid n}$. Then

$$
\delta_{1 \mid n}=\left\langle\delta_{1 \mid n}, e\right\rangle e+\left\langle\delta_{1 \mid n}, \bar{e}\right\rangle \bar{e} .
$$

Now suppose that the unit normal vector field $e_{m+1}$ is parallel in the normal bundle of $M^{m}$, i.e., by the definition, $d e_{m+1}$ is tangent to $M^{m}$ everywhere. Then by choosing $e_{m+1}=e$ and $e_{m+2}=\bar{e}$ everywhere on $M^{m}$ and using (4.15) and (4.16) we obtain

$$
\left\langle\delta_{1}, e_{m+\alpha}\right\rangle=0, \quad(\alpha=3, \cdots, n-m),
$$

and therefore

$$
(V)_{\alpha}=0, \quad(3 \leq \alpha \leq n-m) .
$$

Combination of (4.4), (4.11), .., (4.14), (4.18) gives

$$
\begin{array}{r}
d\left(\sqrt{a}|\delta_{1}, \underbrace{d x, \cdots, d x}_{m-i}, \underbrace{d e_{m+1}, \cdots, d e_{m+1}}_{i-1}, e_{m+1}, \cdots, e_{n}|\right) \\
=(-1)^{i-1} m !\left[\rho K_{i-1}\left(e_{m+1}\right)+\left\langle\delta_{1}, e_{m+1}\right\rangle K_{i}\left(e_{m+1}\right)+F_{i}\left(e_{m+1}\right)\right] d V \\
(i=1, \cdots, m) .
\end{array}
$$

where

$$
\begin{array}{r}
F_{i}\left(e_{m+1}\right)=\frac{(m-i) !(i-1) !}{m !}\left\langle\delta_{1}, e_{m+2}\right\rangle \sum_{j_{m-i+1}, \cdots, j_{m-1}} k_{j_{m-i+1}} \\
\cdots k_{j_{m-1}} \Omega_{m+2 \mid} j_{m} g^{k j_{m}}
\end{array}
$$

Integrating (4.19) over an oriented $M^{m}$ and applying Stokes' theorem we hence arrive at 
Theorem 4.1. Let $x: M^{m} \rightarrow N^{n}$ be a closed oriented m-dimensional $(2 \leq m<n)$ submanifold of class $C^{3}$ immersed in a Riemannian n-manifold $N^{n}$, which is of constant sectional curvature and admits a continuous infinitesimal conformal vector field $\xi$. If $e_{m+1}$ and $e_{m+2}$ are unit normal vector fields over $M^{m}$ such that $e_{m+1}$ is parallel in the normal bundle of $\boldsymbol{M}^{m}$, and $e_{m+1}, e_{m+2}$ are coplanar with the normal component of $\xi$, then

$$
\begin{aligned}
\int_{M^{m}}\left[\rho K_{i-1}\left(e_{m+1}\right)+\left\langle\delta, e_{m+1}\right\rangle K_{i}\left(e_{m+1}\right)\right] d V=-\int_{M^{m}} F_{i}\left(e_{m+1}\right) d V, \\
(i=1, \cdots, m),
\end{aligned}
$$

where $\rho$ is given by (3.32).

Remarks. 1. If $n-m=1$, then $F_{i}\left(e_{m+1}\right)=0, i=1, \cdots, m$, hold automatically, and formulas (4.21) are due to Hsiung [5] for Euclidean $N^{n}$ with $\xi$ generated by the position vector $x$ of a general point of $M^{m}$ with respect to a fixed point 0 in $N^{n}$, due to Hsiung [6] and Feeman and Hsiung [3] for a Riemannian $N^{n}$ and a special $\xi$, and due to Katsurada [7] for a Riemannian $N^{n}$ and a general $\xi$.

2. For Euclidean $N^{n}$ and general $n$ with the position vector field $x$ as $\xi$, formulas (4.21) are due to Chen and Yano [1], and due to Yano [14], [15] under some additional conditions.

3. For Euclidean $N^{n}$, the condition of the parallelism of $e_{m+1}$ in the normal bundle of $M^{m}$ can be replaced by the condition that $M^{m}$ be immersed in a hypersphere of $N^{n}$ centered at the origin of $N^{n}$.

4. For a special $e_{m+1}$, formulas (4.21) are due to Katsurada and Kôjyô [13], and Katsurada [8].

\section{Characterizations of umbilical submanifolds}

In this section we use integral formula (4.21) to derive various conditions for a submanifold of a Riemannian manifold to be umbilical with respect to a given normal vector field. For this purpose we first state the following three lemmas which will be needed for the proofs of our main theorems. The proofs of the lemmas are omitted here, but can be found in [4, pp. 52, 104-105].

Lemma 5.1. Let $K_{i}\left(e_{A}\right), i=1, \cdots, m$, be given by (3.23). Then

$$
K_{i}\left(e_{A}\right)^{2}-K_{i-1}\left(e_{A}\right) K_{i+1}\left(e_{A}\right) \geq 0, \quad(i=1, \cdots, m-1),
$$

where the equality implies that $k_{1}\left(e_{A}\right)=\cdots=k_{m}\left(e_{A}\right)$.

Lemma 5.2. If $K_{i}\left(e_{A}\right), K_{i-1}\left(e_{A}\right), \cdots, K_{i-j-1}\left(e_{A}\right)>0,1 \leq j<i \leq m$, then

$$
\frac{K_{i-1}\left(e_{A}\right)}{K_{i}\left(e_{A}\right)} \geq \frac{K_{i-2}\left(e_{A}\right)}{K_{i-1}\left(e_{A}\right)} \geq \cdots \geq \frac{K_{i-j-1}\left(e_{A}\right)}{K_{i-j}\left(e_{A}\right)},
$$


where the equality at any stage implies that $k_{1}\left(e_{A}\right)=\cdots=k_{m}\left(e_{A}\right)$.

Lemma 5.3. If $K_{1}\left(e_{A}\right), \cdots, K_{j}\left(e_{A}\right)>0, j \leq m$, then

$$
K_{1}\left(e_{A}\right) \geq K_{2}\left(e_{A}\right)^{1 / 2} \geq K_{3}\left(e_{A}\right)^{1 / 3} \geq \cdots \geq K_{j}\left(e_{A}\right)^{1 / j},
$$

where the equality at any stage implies that $k_{1}\left(e_{A}\right)=\cdots=k_{m}\left(e_{A}\right)$.

In the remainder of this section we shall use the following notation:

$N^{n}$ : A Riemannian $n$-manifold $(n>2)$ having constant sectional curvature and admitting a continuous infinitesimal conformal vector field $\xi$ so that $L_{\xi} a_{\alpha \beta}=2 \rho a_{\alpha \beta}$ where $a_{\alpha \beta}$ is the Riemannian metric tensor of $N^{n}$.

$M^{m}: A$ closed oriented $m$-dimensional $(n>m \geq 2)$ submanifold of class $C^{3}$ immersed in $N^{n}$.

$e$ : A unit normal vector field on $\boldsymbol{M}^{m}$ parallel in the normal bundle of $\boldsymbol{M}^{m}$. $k_{i}, K_{i}, F_{i}$, and $p: \quad k_{i}(e), K_{i}(e), F_{i}(e)$ for $i=1, \cdots, m$, and $\langle\xi, e\rangle$, respectively.

Theorem 5.1. $M^{m}$ is umbilical with respect to $e$ if at all points of $M^{m}$ for an integer or $i, 1 \leq i \leq m$,

(i) $\rho / K_{i}>0$,

(ii) $p \leq-\rho K_{i-1} / K_{i}$ (or $\left.p \geq-\rho K_{i-1} / K_{i}\right)$,

(iii) $F_{i}=F_{i+1}=0$ for $1 \leq i<m$, and $F_{i}=F_{i-1}=0$ for $i=m$.

For Euclidean $N^{n}$ with the position vector field $x$ as $\xi$, Theorem 5.1 is due to Hsiung [5] for $n=m+1$ and due to Chen and Yano [1] for general $n$ and $2<i<m$. For Riemannian $N^{n}$ with a special $e$ and $i=1$, Theorem 5.1 is due to Katsurada [8].

Proof. By (ii), the integrand of (4.21) for $e_{m+1}=e$ is either nonpositive or nonnegative, and therefore we have

$$
p=\rho K_{i-1} / K_{i}
$$

For $i<m$, substituting (5.4) in (4.21), where $i$ is replaced by $i+1$, gives

$$
\int_{M^{m}} \frac{\rho}{K_{i}}\left(K_{i}^{2}-K_{i-1} K_{i+1}\right) d V=0
$$

Due to (i) and (5.1), the integrand of (5.5) is nonnegative, and therefore (5.5) holds only when, at all points of $M^{m}, K_{i}{ }^{2}-K_{i-1} K_{i+1}=0$. From Lemma 5.1 it follows that $k_{1}=\cdots=k_{m}$ at all points of $M^{m}$, and hence $M^{m}$ is umbilical with respect to $e$.

For $i=m$, substituting (5.4) in (4.21) where $i$ is replaced by $i-1$, we obtain

$$
\int_{M^{m}} \frac{\rho}{K_{m}}\left(K_{m-1}^{2}-K_{m-2} K_{m}\right) d V=0
$$


By applying Lemma 5.1 with the same argument as above, we can show that $M^{m}$ is also umbilical with respect to $e$.

Theorem 5.2. $M^{m}$ is umbilical with respect to $e$ if at all points of $M^{m}$ for an integer $i, 1<i<m$,

(i) $\rho, K_{i+1}, K_{i}, K_{i-1}>0$,

(ii) $p \geq-\rho K_{i-1} / K_{i}$,

(iii) $F_{i+1}=0$.

For Euclidean $N^{n}$ with the position vector field $x$ as $\xi$, Theorem 5.2 is due to Chen and Yano [1]. It should be remarked that we may have a similar theorem by assuming $\rho<0$ instead of $\rho>0$.

Proof. By (ii) and Lemma 5.2 we have

$$
p \geq-\rho K_{i-1} / K_{i} \geq-\rho K_{i} / K_{i+1} .
$$

(4.21), with $i$ replaced by $i+1$, and (5.7) imply that the equality holds in (5.7), and hence $M^{m}$ is umbilical with respect to $e$ by Lemma 5.2.

Theorem 5.3. $M^{m}$ is umbilical with respect to $e$ if at all points of $M^{m}$ for an integer $s, 1 \leq s \leq m$,

(i) $p$ is of the same sign,

(ii) $K_{i}>0, i=1, \cdots, s$,

(iii) $K_{s}$ is constant,

(iv) $\rho$ is of the same sign,

(v) $F_{1}=F_{s+1}=0$ for $1<s<m$, and $F_{1}=F_{s}=0$ for $s=m$.

For Euclidean $N^{n}$ and $n=m+1$, Theorem 5.3 is due to Hsiung [5].

Proof. Case 1. $s<m$. By (ii) and inequality (5.1) for $i=1, \cdots, s$ we obtain

$$
K_{1} / K_{0} \geq K_{2} / K_{1} \geq \cdots \geq K_{s+1} / K_{s}
$$

and, in particular,

$$
K_{1} K_{s} \geq K_{s+1}
$$

where the equality holds only when $k_{1}=\cdots=k_{m}$ in view of Lemma 5.1. Here we assume $\rho>0$. Then from (4.21) for $i=1$ and assumptions (i), (ii), (v) it follows that $p$ is negative. (For the case $\rho<0$, the arguments in the proof of our theorem will be exactly the same, except that $p$ would be positive.) Multiplying both sides of inequality (5.8) by $p$, integrating over $M^{m}$, and applying (4.21) for $i=1$ and $i=s+1$, we can readily obtain, in consequence of (iii) and (v),

$$
-\int_{M^{m}} \rho K_{s} d V=\int_{M^{m}} p K_{1} K_{s} d V \leq \int_{M^{m}} p K_{s+1} d V=-\int_{M^{m}} \rho K_{s} d V,
$$

from which it follows that 


$$
\int_{M m} p\left(K_{1} K_{s}-K_{s+1}\right) d V=0 .
$$

Since $p$ is negative, from (5.8) we see that the integrand in (5.9) is nonpositive and therefore must be zero. Thus the equality holds in (5.8) so that $k_{1}=\cdots$ $=k_{m}$ everywhere by Lemma 5.1. Hence $M^{m}$ is umbilical with respect to $e$.

Case 2. $s=m$. From (ii), (iii) and Lemma 5.3 it follows that

$$
K_{1} \geq K_{2}^{1 / 2} \geq \cdots \geq K_{m-1}^{1 /(m-1)} \geq K_{m}^{1 / m}=c,
$$

where $c$ is a positive constant. By means of (4.21) for $i=m$, assumption (v) and inequalities (5.10), we obtain

$$
\int_{M^{m}} p K_{m} d V=-\int_{M^{m}} \rho K_{m-1} d V<-c^{m-1} \int_{M^{m}} \rho d V .
$$

On the other hand, using (4.21) for $i=1,(v),(5.10)$ and the fact that $p<0$, we have

$$
\begin{aligned}
\int_{M^{m}} p K_{m} d V & =\int_{M^{m}} p c^{m} d V=c^{m-1} \int_{M^{m}} p K_{m}^{1 / m} d V \\
& \geq c^{m-1} \int_{M^{m}} p K_{1} d V=-c^{m-1} \int_{M^{m}} \rho d V .
\end{aligned}
$$

Combination of (5.11) and (5.12) shows immediately that the equality holds in (5.12) and therefore that

$$
\int_{M m} p\left(K_{m}^{1 / m}-K_{1}\right) d V=0 .
$$

Since $p<0,(5.10)$ implies that the integrand of (5.13) is nonnegative and therefore that $K_{1}=K_{m}^{1 / m}$. Thus by Lemma $5.3, k_{1}=\cdots=k_{m}$ at all points of $M^{m}$. Hence the proof of Theorem 5.3 is complete.

Theorem 5.4. $M^{m}$ is umbilical with respect to $e$ if at all points of $M^{m}$ for two integers $i$ and $s, i \leq i<s \leq m$,

(i) $\boldsymbol{K}_{i}, \boldsymbol{K}_{i+1}, \cdots, \boldsymbol{K}_{s}>0$,

(ii) $K_{s}=\sum_{j=1}^{s-1} c_{j} K_{j}$, for some constants $c_{j} \geq 0, i \leq j \leq s-1$,

(iii) $\rho$ is of the same sign,

(iv) $F_{j}=0, j=1, \cdots, s-1$.

Proof. We observe

$$
\frac{K_{j}}{K_{s}}-\frac{K_{j-1}}{K_{s-1}}=\frac{K_{j}}{K_{s-1}}\left(\frac{K_{s-1}}{K_{s}}-\frac{K_{j-1}}{K_{j}}\right) .
$$

In view of Lemma 5.2, the right side of (5.14) is nonnegative for $i \leq j \leq s-1$. Thus 


$$
K_{j} / K_{s} \geq K_{j-1} / K_{s-1}
$$

where the equality holds only when $k_{1}=\cdots=k_{m}$. By (ii) and (5.15) we obtain

$$
1=\sum_{j=i}^{s-1} c_{j} K_{j} / K_{s} \geq \sum_{j=i}^{s-1} c_{j} K_{j-1} / K_{s-1}
$$

or

$$
K_{s-1}-\sum_{j=i}^{s-1} c_{j} K_{j-1} \geq 0
$$

where the equality holds only when $k_{1}=\cdots=k_{m}$. Thus by means of (4.21), (iv) and (ii) we obtain

$$
\int_{M^{m}} \rho\left(K_{s-1}-\sum_{j=i}^{s-1} c_{j} K_{j-1}\right) d V=-\int_{M^{m}} p\left(K_{s}-\sum_{j=i}^{s-1} c_{j} K_{j}\right) d V=0 .
$$

(5.16), (5.17), (iii) show immediately that the equality holds in (5.16). Hence $M^{m}$ is umbilical with respect to $e$.

Theorem 5.5. $M^{m}$ is umbilical with respect to $e$ if at all points of $M^{m}$ for two integers $i$ and $s, 0 \leq i<s<m$,

(i) $K_{i}, \cdots, K_{s+1}>0$,

(ii) $K_{s}=\sum_{j=i}^{s-1} c_{j} K_{j}$, for some constants $c_{j} \geq 0, i \leq j \leq s-1$,

(iii) $p$ is of the same sign,

(iv) $F_{j}=0, j=1, \cdots, s-1$.

Proof. By Lemma 5.2 we have

$$
\frac{K_{j}}{K_{s}}-\frac{K_{j+1}}{K_{s+1}}=\frac{K_{j}}{K_{s+1}}\left(\frac{K_{s+1}}{K_{s}}-\frac{K_{j+1}}{K_{j}}\right) \leq 0,
$$

where the equality holds only when $k_{1}=\cdots k_{m}$. From (ii), (5.18) it follows that

$$
1=\sum_{j=i}^{s-1} c_{j} K_{j} / K_{s} \leq \sum_{j=i}^{s-1} c_{j} K_{j+1} / K_{s+1}
$$

or

$$
K_{s+1}-\sum_{j=i}^{s-1} c_{j} K_{j+1} \leq 0
$$

where the equality holds only when $k_{1}=\cdots=k_{m}$. Thus by means of (4.21), (iv) and (ii) we obtain 


$$
\int_{M^{m}} p\left(K_{s+1}-\sum_{j=i}^{s-1} c_{j} K_{j+1}\right) d V=-\int_{M^{m}} \rho\left(K_{s}-\sum_{j=i}^{s-1} c_{j} K_{j}\right) d V=0 .
$$

(5.19), (5.20), (iii) show immediately that the equality holds in (5.19). Hence $M^{m}$ is umbilical with respect to $e$.

Theorem 5.6. $M^{m}$ is umbilical with respect to $e$ if at all points of $M^{m}$ for an integer $i, 1<i \leq m$,

(i) $K_{i}>0$,

(ii) $K_{i}=c K_{i-1}$, for some constant $c$,

(iii) $\rho$ is of the same sign,

(iv) $F_{i-1}=F_{i}=0$.

Proof. Due to (i), $c$ cannot be zero and $K_{i-1}$ must be of a fixed sign. Using (ii) and Lemma 5.1 we have

$$
K_{i-1}\left(K_{i-1}-c K_{i-2}\right)=K_{i-1}^{2}-K_{i} K_{i-2} \geq 0
$$

so that

$$
K_{i-1}-c K_{i-2} \quad \text { is of fixed sign , }
$$

and vanishes identically only when $k_{1}=\cdots=k_{m}$. Thus by means of (4.21), (iv) and (ii) we obtain

$$
\int_{M^{m}} \rho\left(K_{i-1}-c K_{i-2}\right) d V=-\int_{M^{m}} p\left(K_{i}-c K_{i-1}\right) d V=0
$$

(5.21), (5.22), (iii) imply immediately that $K_{i-1}=c K_{i-2}$. Hence Theorem 5.6 is proved.

Corollary 5.6. $M^{m}$ is umbilical with respect to $e$ if at all points of $M^{m}$

(i) $K_{m}>0$

(ii) $\sum_{i=1}^{m}\left(1 / k_{i}\right)=$ constant,

(iii) $\rho$ is of the same sign,

(iv) $F_{m-1}=F_{m}=0$.

Proof. By (ii) and the definition (3.23) of $K_{i}$ we obtain

$$
m K_{m-1} / K_{m}=\sum_{j=1}^{m}\left(1 / k_{i}\right)=\text { constant }
$$

so that

$$
K_{m}=c K_{m-1}, \quad \text { for some constant } c .
$$

Hence Corollary 5.6 is an immediate consequence of Theorem 5.6 for $i=m$.

Theorem 5.7. $M^{m}$ is umbilical with respect to $e$ if at all points for an integer $s, 1<s \leq m$, and a constant $c$

(i) $K_{i}>0$ for $i=1, \cdots, s$, 
(ii) $K_{s-1}{ }^{1 /(s-1)} \geq c \geq K_{s}{ }^{1 / s}$,

(iii) $p$ is of the same sign,

(iv) $\rho$ is of the same sign,

(v) $F_{i}=F_{2}=F_{s}=0$.

Proof. As in the proof of Theorem 5.3 we may assume $\rho>0$. Then due to (iii), (v) and (i) for $i=1$, (4.21) for $i=1$ implies $p<0$. By (5.3), (ii) we have $K_{1}>K_{s-1}^{1 /(s-1)} \geq c$, and therefore, in consequence of (ii), (4.21) for $i=s$ and $i=1$,

$$
\begin{aligned}
-\int_{M^{m}} c^{s-1} p K_{1} d V & \geq-\int_{M^{m}} c^{s} p d V \geq-\int_{M^{m}} p K_{s} d V \\
& =\int_{M^{m}} \rho K_{s-1} d V \geq \int_{M^{m}} \rho c^{s-1} d V \\
& =-\int_{M^{m}} c^{s-1} p K_{1} d V .
\end{aligned}
$$

Thus the equality holds everywhere in (5.23), so that

$$
\int_{M^{m}} p\left(K_{1}-c\right) d V=0
$$

which implies that $K_{1}=c$. Hence, by Theorem 5.3 for $s=1, M^{m}$ is umbilical with respect to $e$.

Theorem 5.8. $M^{m}$ is umbilical with respect to $e$ if at all points of $M^{m}$ for an integer $s, 1<s \leq m$, and a constant $c$

(i) $K_{s-1}, K_{s}>0$,

(ii) $K_{s-1} / K_{s} \geq c \geq K_{s-2} / K_{s-1}$,

(iii) $p$ is of the same sign,

(iv) $\rho$ is of the same sign,

(v) $F_{s-1}=F_{s}=0$.

Proof. As before we may assume $\rho>0$. Then due to (i), (iii) and (v), (4.21) implies $p<0$. By using (ii), (4.21) for $i=s-1$ and $i=s$ we have

$$
\begin{aligned}
\int_{M^{m}} \rho K_{s-2} d V & =-\int_{M^{m}} p K_{s-1} d V \geq-\int_{M^{m}} c p K_{s} d V \\
& =\int_{M^{m}} c \rho K_{s-1} \geq \int_{M^{m}} \rho K_{s-2} d V .
\end{aligned}
$$

Thus the equality holds everywhere in (5.24), so that

$$
\int_{M^{m}} p\left(K_{s-1}-c K_{s}\right) d V=0
$$

Since $p\left(K_{s-1}-c K_{s}\right) \leq 0,(5.25)$ implies that $K_{s-1}=c K_{s}$ at all points of $M^{m}$. Hence, by Theorem 5.6 for $i=s, M^{m}$ is umbilical with respect to $e$. 
Theorems 5.4, 5.5, 5.6 and Corollary 5.6 are due to Chen and Yano [1] for Euclidean $N^{n}$ with the position vector field $x$ as $\xi$. Theorems $5.4, \cdots, 5.8$ are due to Strong [12] for $n=m+1$ with the position vector field $x$ as $\xi$.

\section{References}

[1] B. Y. Chen \& K. Yano, Integral formulas for submanifolds and their applica. tions, J. Differential Geometry 5 (1971) 467-477.

[2] L. P. Eisenhart, Riemannian geometry, Princeton University Press, Princeton, 1949.

[3] G. F. Feeman \& C. C. Hsiung, Characterizations of Riemann n-spheres, Amer. J. Math. 81 (1959) 691-708.

[ 4 ] G. H. Harảy, J. E. Littlewood \& G. Pólya, Inequalities, Cambridge University Press, Cambridge, 1934.

[5] C. C. Hsiung, Some integral formulas for closed hypersurfaces, Math. Scand. 2 (1954) 286-294.

[6] - Some integral formulas for closed hypersurfaces in Riemannian space, Pacific J. Math. 6 (1956) 291-299.

[7] Y. Katsurada, Generalized Minkowski formulas for closed hypersurfaces in Riemann space, Ann. Mat. Pura Appl. 57 (1962) 283-293.

[8] - Closed submanifolds with constant ע-th mean curvature related with a vector field in a Riemannian manifold, J. Fac. Sci. Hokkaido Univ. Ser. I, 20 (1969) $171-181$.

[9] Y. Katsurada \& H. Kôjyô, Some integral formulas for closed submanifolds in a Riemann space, J. Fac. Sci. Hokkaido Univ. Ser. I, 20 (1968) 90-100.

[10] Y. Katsurada \& T. Nagai, On some properties of a submanifold with constant mean curvature in a Riemann space, J. Fac. Sci. Hokkaido Univ. Ser. I, 20 (1968) 79-89.

[11] H. Minkowski, Volumen und Oberfäche, Math. Ann. 57 (1903) 447-495.

[12] R. E. Stong, Some characterizations of Riemann n-spheres, Proc. Amer. Math. Soc. 11 (1960) 945-51.

[13] A. W. Tucker, On generalised covariant differentiation, Ann. of Math. 32 (1931) 451-460.

[14] K. Yano, Integral formulas for submanifolds and their applications, Canad. J. Math. 22 (1970) 376-388.

[15] - Submanifolds with parallel mean curvature vector of a Euclidean space or a sphere, Kōdai Math. Sem. Rep. 23 (1971) 144-159.

[16] C. E. Weatherburn, An introduction to Riemannian geometry and the tensor calculus, Cambridge University Press, Cambridge, 1950.

\section{LEHIGH UNIVERSITY \\ National Tsing Hua University \\ and ACAdemia Sinica \\ Pennsylvania Bureau of Correction}


